\title{
Development of the Self-Powered Extravehicular Mobility Unit Extravehicular Activity Data Recorder
}

\author{
Craig Bernard ${ }^{1}$ and Terry R. Hill ${ }^{2}$ \\ NASA Johnson Space Center, Houston, TX, 77058 \\ Murray, Sean ${ }^{3}$ \\ Hamilton Sundstrand, Windsor Locks, CT, 06095 \\ Wichowski, Robert ${ }^{4}$ \\ Hamilton Sundstrand, Windsor Locks, CT, 06095 \\ and \\ Rosenbush, David ${ }^{5}$ \\ Hamilton Sundstrand, Windsor Locks, CT, 06095
}

\begin{abstract}
The Self-Powered Extravehicular Mobility Unit (EMU) Extravehicular Activity (EVA) Data Recorder (SPEEDR) is a field-programmable gate array (FPGA)-based device designed to collect high-rate EMU Primary Life Support Subsystem (PLSS) data for download at a later time. During EVA, the existing EMU PLSS data downlink capability is one data packet every 2 minutes and is subject to bad packets or loss of signal. Higher-rate PLSS data is generated by the Enhanced Caution and Warning System but is not normally captured or distributed. Access to higher-rate data will increase the capability of EMU anomaly resolution team to pinpoint issues remotely, saving crew time by reducing required call-down Q\&A and on-orbit diagnostic activities. With no Space Shuttle flights post Fiscal Year 2011 (FY11), and potentially limited down-mass capability, the ISS crew and ground support personnel will have to be capable of on-orbit operations to maintain, diagnose, repair, and return to service EMU hardware, possibly through 2028. Collecting high-rate EMU PLSS data during both intravehicular activity (IVA) and EVA operations will provide trending analysis for life extension and/or predictive performance. The SPEEDR concept has generated interest as a tool/technology that could be used for other International Space Station subsystems or future exploration-class space suits where hardware reliability/availability is critical and low/variable bandwidth may require "store then forward" methodology. Preliminary work in FY11 produced a functional prototype consisting of an FPGA evaluation board, custom memory/interface circuit board, and custom software. The SPEEDR concept includes a stand-alone battery that is recharged by a computer Universal Serial Bus (USB) port while data are being downloaded.
\end{abstract}

\section{Nomenclature}

CSV $=$ Comma Separated Values

ECWS $=$ Enhanced Caution and Warning System

${ }^{1}$ EMU Portable Life Support System Subsystem Manager, Crew and Thermal Systems Division, 2101 NASA Parkway, Houston, Texas 77058 Mail Stop EC5.

2 EMU Subsystem Sustainability through 2028 Engineer, Crew and Thermal Systems Division, 2101 NASA

Parkway, Houston, Texas 77058/ Mail Stop EC5.

${ }^{3}$ Electronics Guru - add your own title/description

${ }^{4}$ FPGA Guru - add your own title/description

${ }^{5}$ Software Guru - add your own title/description 


$\begin{array}{ll}\text { EMU } & =\text { Extravehicular Mobility Unit } \\ \text { EVA } & =\text { extravehicular activity } \\ \text { FPGA } & =\text { field-programmable gate array } \\ \text { FY11 } & =\text { Fiscal Year } 2011 \\ \text { ISS } & =\text { International Space Station } \\ \text { IVA } & =\text { intravehicular activity } \\ \mathrm{O}_{2} & =\text { oxygen } \\ \text { PLSS } & =\text { Portable Life Support Subsystem } \\ \text { SCU } & =\text { Service and Cooling Umbilical } \\ \text { SPEEDR } & =\text { Self-Powered Extravehicular Mobility Unit Extravehicular Activity Data Recorder } \\ \text { STS } & =\text { Space Transportation System } \\ \text { USB } & =\text { Universal Serial Bus }\end{array}$

\section{Introduction}

$\mathrm{T}$ The Self-Powered Extravehicular Mobility Unit (EMU) Extravehicular Activity (EVA) Data Recorder (SPEEDR) was developed through a collaborative effort between NASA's Johnson Space Center and Hamilton Sundstrand (Windsor Locks, CT) to aid in real-time mission support for the International Space Station (ISS) and Space Shuttle EMU space suit by recording higher-rate Primary Life Support System (PLSS) data.

The PLSS has 14 analog and 10 discrete signals that are received and analyzed by the Enhanced Caution and Warning System (ECWS). The ECWS also transmits the data to two serial data ports: a 50-baud port at a rate4 of 1 record per 20 seconds; and a 4800-Baud port at a rate of 7 records per second. The lower-rate 50-baud port is connected to the radio to provide real-time data distribution to the ground. This radio channel is shared with the Biomed data during EVA operations, such that Portable Life Support Subsystem (PLSS) data are transmitted only once every 2 minutes. The higher-rate 4800-Baud port is used during ground-based lab and chamber activities, but is not used during on-orbit EVA operations. The SPEEDR concept will allow the capture of the higher-rate 4800-baud port data for later download, during both intravehicular (IVA) and EVA operations. The design essentially offers a "Black Box" recording capability. A block diagram of the concept is shown below in figure 1 .

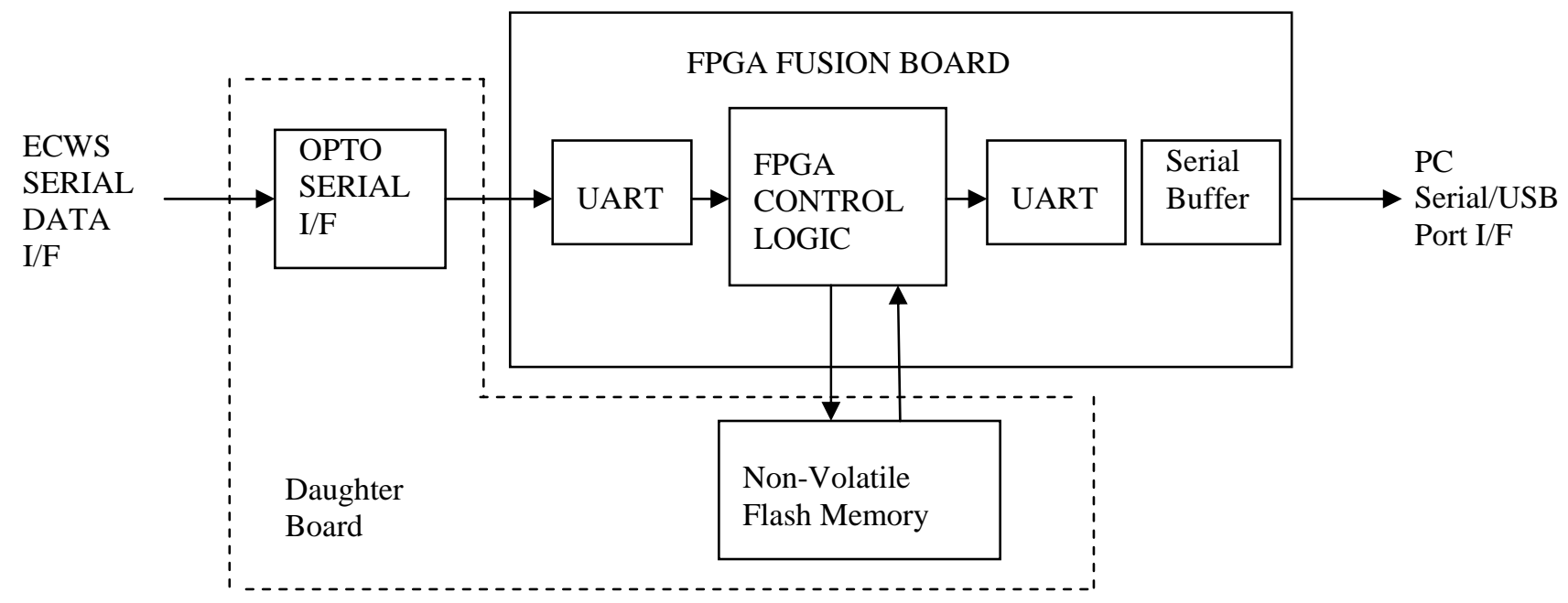

Figure 1: SPEEDR Conceptual Block Diagram

\section{Benefits to Obtaining Higher-Rate Extravehicular Mobility Unit Data}

A sample rate of 8.3 millihertz allows for many events to occur between samples. A good example of this occurred at the end of Space Transportation System (STS)-132 EVA \#3. When the second crew member connected his Service and Cooling Umbilical, the first crew member reported a "high $\mathrm{O}_{2}$ use" message. The pressure drop from the second connection, coupled with temperature fluxuations of the Shuttle supply tanks, could have briefly 
caused a pressure rate drop in the system sufficient enough to trigger that message. Without higher-rate data, only a plausible theory remained.

Not only would anomaly resolution efforts benefit from higher-rate data, trending analysis for the PLSS would be enhanced as we proceed into the post-Shuttle era of space flight. Previously, the maximum time a PLSS would remain on the International Space Station (ISS) was approximately 2 years, at which point the PLSS would be returned to the ground for refurbishment. We have recently developed a "mega-dizing" process to provide a 6-year on-orbit life certification. Having never operated in this mode before, we realize that there are a number of unknowns of which higher-rate data could provide trending analysis. Data recorded during IVA operations, such as with the Airlock Cooling Loop Recovery Unit (A/L CLR) and during suit check outs and crew fit checks, would also assist in the trending analysis.

\section{Concept}

The initial concept behind the baseline SPEEDR was developed to keep the device simple and stand-alone, and to minimize perturbation to the both the EMU hardware and the on-orbit operations. The device would be selfpowered, with the battery and memory being sized for 1 year of unattended use. The data recorder will operate at very low-level standby power state, wake up about every 5 minutes, look for activity on the data bus, and begin recording data when activity is detected. Battery recharge would occur via the Universal Serial Bus (USB) port while the data are being accessed, thereby eliminating the need for another battery charging system. Connection to the existing cable harness would take place in the same area where the battery and the Contamination Control Cartridge (CCC) are frequently accessed. One possible deisign solution is FPGA based which would minimize complexity and increase reliability by reducing the overall EEE part count.

The SPEEDR can be broken down into three parts: subsystem interface; data storage; and ISS data system interface. The subsystem interface includes the connector, wires, and protocol needed to communicate with the unique hardware. The data storage includes the memory necessary to record the parameters for later download. The ISS data system interface provides a method to transmit the data to the ground personnel via the ISS. In this case, the subsystem is the EMU PLSS ECWS, the memory is nonvolatile flash, and the ISS data system interface is the USB port on the ISS laptop.

The SPEEDR subsystem interface could be modified to create other versions of the SPEEDR to interface with different ISS subsystems that provide a serial data diagnostic test port. Some potential EMU-related ISS subsystems with a serial test port include the METOX regenerator, the LIB charger, and the ISS Simplified Aid for EVA Rescue (currently in development). These subsystems have data interfaces that are not, or will not be, used on-orbit regularly due to time constraints or equipment availability. Having access to those data would prove beneficial during occasional anomalies, missed call-downs, or on-orbit checkouts/calibrations. The other two parts of the SPEEDR, data storage and ISS data system interface, could be reused "as is" for these other subsystems. Some ISS subsystems were developed to interface with the older ISS laptop RS-485 serial data interface, and have had problems with the USB adapters and their associated part specific software drivers used to interface with the newer ISS laptop computers. Having a proven ISS data system interface could have benefit for these subsystems and minimize additional specialized software and drivers and their associated certifications for use on ISS. SPEEDR knows about the data which it is receiving. This allows us to put a timestamp and battery data into every record. Specific logic is required for each new type data stream. Reuse "as-is" can be achieved by changing to logic which is more like a tape recorder. This would weaken the linkage between time stamps and the data. It would also be a significant change to the FPGA logic. SPEEDR would still have to be told the serial port configuration, since various hardware protocols and baud rates etc are used.

This same "store then forward" concept is relevant to future space exploration scenarios where bandwidth is limited and variable.

\section{Prototype Development}

The initial bread board hardware design is based on an Actel Fusion FPGA Embedded development board. The use of the Fusion board facilitated rapid development of the detailed Very High Speed Integrated Circuits (VHSIC) Hardware Description Language (VHDL). This VHDL is ported into the FPGA device and controls the serial data interface to the EMU data port, the storage of the data in the memory, the various Sleep and Wake modes for power conservation, and the download of the data when attached to a PC USB port. The same VHDL code can be ported into a radiation-tolerant version of an Actel FPGA device for the flight design, which will be optimized for the battery version of this design. 
The benefits of an FPGA based design are reduced overall complexity, lower part count, and some immunity to EEE parts obsolescence. As an FPGA vendor changes their part types and families over the years, the same VHDL code can be ported into new part families with minimal modification. A different type of configuration and quality control is required of the FPGA based VHDL code than that of a classical microprocessor, software based system.

The daughter board contains an optical isolator and nonvolatile memory which was developed to interface directly with digital ports of the FPGA fusion board. The nonvolatile memory is a FLASH NAND based Electrically Erasable Programmable Read-Only Memory (EEPROM). The interface provides the control lines and multiple serial interfaces for data and command. The device is a Toshiba TC58NVG03ETA00, 1-gigabit (128M x 8 -bit) memory chip. The SPEEDR hardware will connect to the opto-isolated 4800 Baud serial port of the ECWS. It can automatically append data to the data in storage, when serial data is detected on this port.

The NAND Flash technology was selected due to increased memory density versus NOR based flash memory. Typically, NAND flash requires $60 \%$ the area of NOR flash and capacities of 1 GBIT are common. The SPEEDR application requires the data to be collected and stored until the device is filled, greater than 1 GBIT, then the data is downloaded for analysis. This is similar to typical industry NAND flash applications, digital camera, cell phone memory, ect. Typical, NOR flash applications require continuous reading of the memory, such as software program execution.

Communication software was developed to download the binary file from the SPEEDR memory and convert the data to Comma Separated Values (CSV) format. When the user is ready to download the data from the SPEEDR system, a USB cable connection will be established between the SPEEDR system and a Microsoft Windows based PC, and the SPEEDR system will begin transmitting data (either by a button, or a command) at a known rate. The CSV-style data structure is easily read by popular applications such as Excel or Math Lab for post processing and the generation of plots, and for detailed analysis of the EMU's operation during the EVA. The PC will run a version of the PCeCWS software, which allows storing the received data as a CSV formatted file, which Microsoft Excel (and other spreadsheet programs) can read. The software can also provide a real-time-like display (at about 10 times real-time speed) of the data from a SPEEDR file.

There were various complexities and innovations that were noted during the implementation of this development effort. The SPEEDR FPGA design needed a hardware solution that was capable of parsing digital data and controlling a Flash Memory device typically accomplished with software complied on a microprocessor. The challenging processes were decoding a serial interface from the ECWS, validating the ECWS data block, formatting the SPEEDR data block, and providing a serial Flash Memory controller for storage of the data block. The SPEEDR data block includes ECWS Data, Real Time Clock time tag, and SPEEDR battery performance data. The most challenging process to perform was creating the Flash Memory controller. The Flash Memory controller needed to be capable of self detecting the next available writeable page address during power initialization. Provide all READ, WRITE, PROGRAM, and ERASE signals to the flash memory. It also needed to provide capacity data to the USB interface for use during download.

\section{Future Development Plans}

Baseline SPEEDR development is continuing, including plans to package into a prototype configuration, with the smallest form factor. Packaging will include the ability to mate/demate direct-current power sources via a dedicated power connector with a USB power bus pass through. This pass through would accommodate battery module connection and recharging. Development plans also include incorporating the ability to update the firmware of the SPEEDR. This would occur when the SPEEDR is connected to a PC laptop computer via a second USB port utilizing an Actel proprietary dongle. This iteration will facilitate use in the labs with flight hardware.

It is desired to begin prototype development of a second-generation SPEEDR that would include WiFi capability. This would allow data transfer without crew involvement. WiFi areas exist both internally and externally on the ISS. Data transfer could wait until the crew member returns to the airlock, or be enabled during EVA. Data transfer during EVA would occur any time the traverse coincides with a "hot spot." The increase in power draw associated with WiFi capability would probably necessitate tapping into the EMU power system, most likely the new Rechargeable EVA Battery Assembly (REBA) battery and cable system, which will power the glove heaters, wireless video system, and helmet lights. With IVA use and applications to other subsystems, a more generic acronym for the SPEEDR might be: Self Percipient Electronically Erasable Data Recorder. 


\section{Conclusion}

A conclusion section is not required, though it is preferred. Although a conclusion may review the main points of the paper, do not replicate the abstract as the conclusion. A conclusion might elaborate on the importance of the work or suggest applications and extensions.

\section{Appendix}

An appendix, if needed, should appear before the acknowledgements.

\section{Acknowledgments}

The "acknowledgments" section if desired.

\section{References}

